\title{
Convex Variational Bayesian Inference for Large Scale Generalized Linear Models
}

\section{Hannes Nickisch ${ }^{1}$ and Matthias W. Seeger ${ }^{2}$}

${ }^{1}$ Max Planck Institute for Biological Cybernetics Tübingen, Germany ${ }^{2}$ Saarland University, Saarbrücken, Germany

\author{
June 16, 2009
}
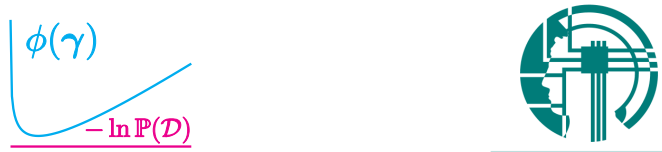


\section{Motivation}

- Image acquisition in MRI
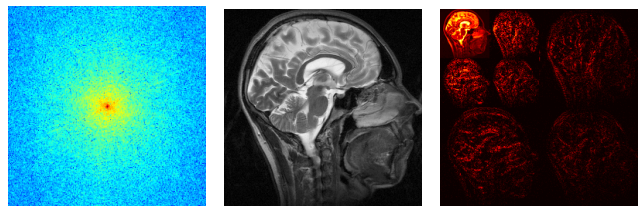

- Binary classification 


\section{Motivation}

- Image acquisition in MRI
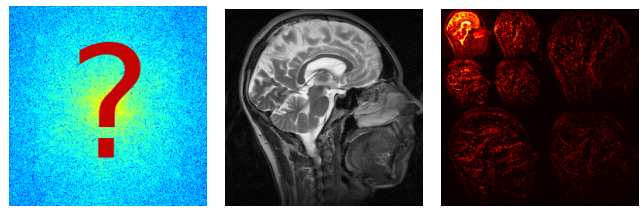

- Binary classification 


\section{Motivation}

- Image acquisition in MRI
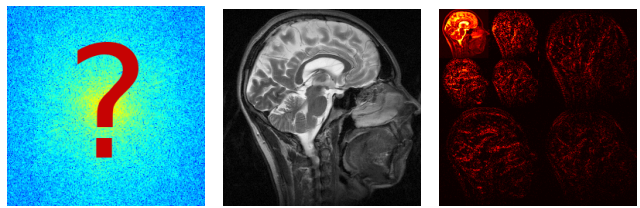

- Binary classification

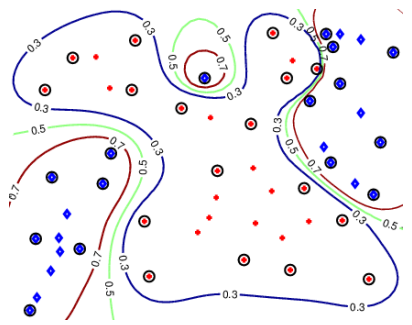




\section{Motivation}

- Image acquisition in MRI
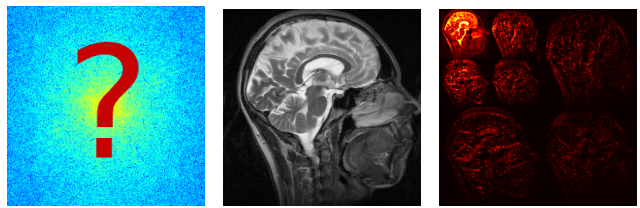

- Binary classification

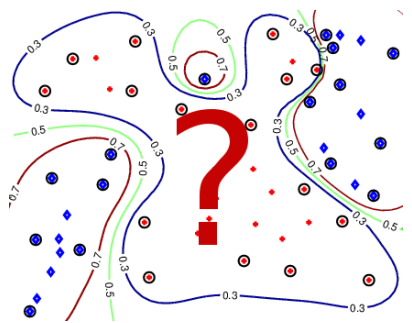




\section{Generalized Linear Model and Experimental Design}

- Generalized Linear Model of $\mathbf{y}=\mathbf{X} \mathbf{u}+\varepsilon, \mathbf{s}=\mathbf{B u}$

- Gaussian $\mathcal{N}\left(r_{i} \mid y_{i}, \sigma^{2}\right)$ and non-Gaussian potentials $t_{j}\left(s_{j}\right)$

$\mathbb{P}(\mathbf{u} \mid \mathcal{D}) \propto \mathcal{N}\left(\mathbf{y} \mid \mathbf{X} \mathbf{u}, \sigma^{2} \mathbf{I}\right) \prod_{j=1}^{n} t_{j}\left(s_{j}\right)$

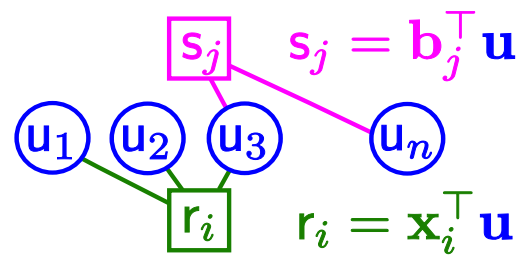




\section{Generalized Linear Model and Experimental Design}

- Generalized Linear Model of $\mathbf{y}=\mathbf{X} \mathbf{u}+\varepsilon, \mathbf{s}=\mathbf{B u}$

- Gaussian $\mathcal{N}\left(r_{i} \mid y_{i}, \sigma^{2}\right)$ and non-Gaussian potentials $t_{j}\left(s_{j}\right)$

$\mathbb{P}(\mathbf{u} \mid \mathcal{D}) \propto \mathcal{N}\left(\mathbf{y} \mid \mathbf{X} \mathbf{u}, \sigma^{2} \mathbf{I}\right) \prod_{j=1}^{n} t_{j}\left(s_{j}\right)$

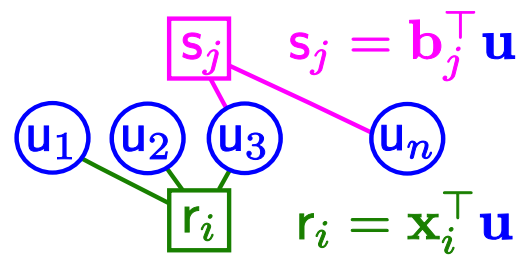

Experimental Design / Measurement Optimization

Along which $\mathbf{x}_{i}$ or $\mathbf{b}_{j}$ shall I measure? $\Rightarrow$ Needs posterior covariance info! 


\section{Generalized Linear Model and Experimental Design}

- Generalized Linear Model of $\mathbf{y}=\mathbf{X} \mathbf{u}+\varepsilon, \mathbf{s}=\mathbf{B u}$

- Gaussian $\mathcal{N}\left(r_{i} \mid y_{i}, \sigma^{2}\right)$ and non-Gaussian potentials $t_{j}\left(s_{j}\right)$

$\mathbb{P}(\mathbf{u} \mid \mathcal{D}) \propto \mathcal{N}\left(\mathbf{y} \mid \mathbf{X} \mathbf{u}, \sigma^{2} \mathbf{I}\right) \prod_{j=1}^{n} t_{j}\left(s_{j}\right)$

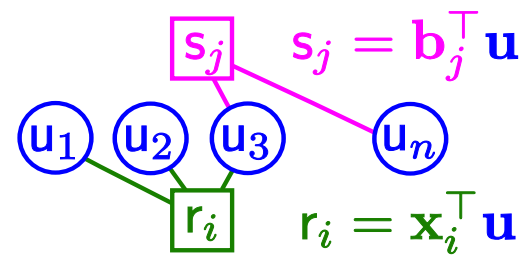

\section{Experimental Design / Measurement Optimization}

Along which $\mathbf{x}_{i}$ or $\mathbf{b}_{j}$ shall I measure? $\Rightarrow$ Needs posterior covariance info!

- MRI: u unknown image

- scanner output $\mathbf{y}$, measurement design $\mathbf{X}$

- sparsity prior $t_{j}\left(s_{j}\right)$ on multi scale gradients $\mathbf{B u}$ 


\section{Generalized Linear Model and Experimental Design}

- Generalized Linear Model of $\mathbf{y}=\mathbf{X} \mathbf{u}+\varepsilon, \mathbf{s}=\mathbf{B u}$

- Gaussian $\mathcal{N}\left(r_{i} \mid y_{i}, \sigma^{2}\right)$ and non-Gaussian potentials $t_{j}\left(s_{j}\right)$

$\mathbb{P}(\mathbf{u} \mid \mathcal{D}) \propto \mathcal{N}\left(\mathbf{y} \mid \mathbf{X} \mathbf{u}, \sigma^{2} \mathbf{I}\right) \prod_{j=1}^{n} t_{j}\left(s_{j}\right)$

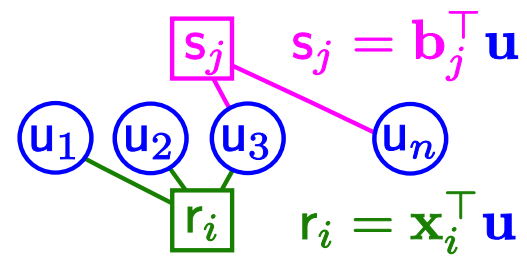

\section{Experimental Design / Measurement Optimization}

Along which $\mathbf{x}_{i}$ or $\mathbf{b}_{j}$ shall I measure? $\Rightarrow$ Needs posterior covariance info!

- Classification: u classifier weights

- Bernoulli potentials and sparsity prior $t_{j}\left(s_{j}\right)$ or Gaussian prior $\mathcal{N}$ on $\mathbf{u}$ 


\section{Posterior I: Site Bounding}

- Legendre-Fenchel (super Gaussian) site bounding:

$$
t_{j}\left(s_{j}\right) \geq \exp \left(\beta_{i} s_{j}-\frac{1}{2} s_{j}^{2} / \gamma_{j}-\frac{1}{2} h\left(\gamma_{j}\right)\right)=: \tilde{t}\left(s_{j}, \gamma_{j}\right)
$$

\section{Laplace}
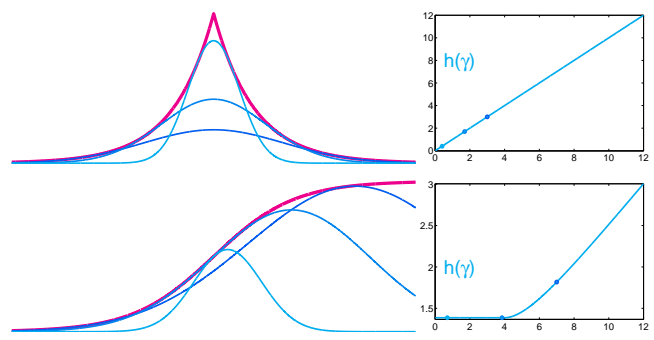

Bernoulli

Student's t
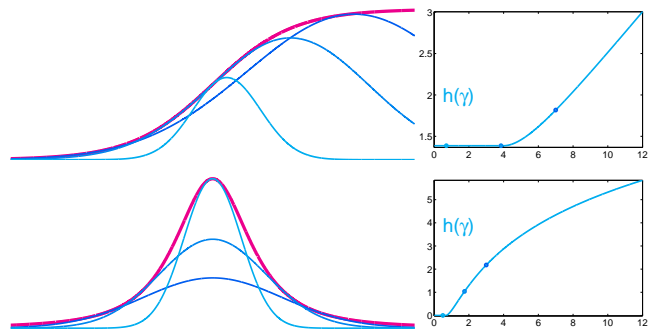


\section{Posterior II: Variational Inference Problem}

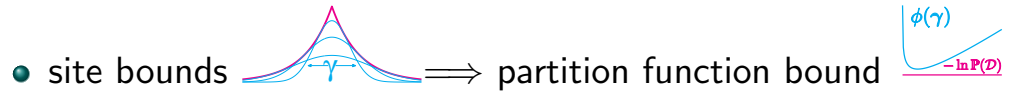

$$
\begin{aligned}
\mathbb{P}(\mathcal{D}) & =\int \mathcal{N}\left(\mathbf{y} \mid \mathbf{X} \mathbf{u}, \sigma^{2} \mathbf{I}\right) \prod_{i=1}^{q} t_{j}\left(s_{j}\right) \mathbf{d} \mathbf{u} \\
& \geq \int \mathcal{N}\left(\mathbf{y} \mid \mathbf{X} \mathbf{u}, \sigma^{2} \mathbf{I}\right) \prod_{i=1}^{q} \tilde{t}\left(s_{j}, \gamma_{j}\right) \mathbf{d} \mathbf{u}=C \cdot \exp \left(-\frac{1}{2} \phi(\gamma)\right) \\
\phi(\gamma) & =\ln \left|\mathbf{A}_{\gamma}\right|+\sum_{j} h_{j}\left(\gamma_{j}\right)+\min _{\mathbf{u}} R(\mathbf{u}, \gamma)
\end{aligned}
$$

$\mathbf{A}_{\gamma}=\mathbf{X}^{\top} \mathbf{X}+\mathbf{B}^{\top} \boldsymbol{\Gamma}^{-1} \mathbf{B}, \quad R(\mathbf{u}, \gamma)=\|\mathbf{X} \mathbf{u}-\mathbf{y}\|^{2}+\mathbf{u}^{\top} \mathbf{B}^{\top} \Gamma^{-1} \mathbf{B} \mathbf{u}-2 \boldsymbol{\beta}^{\top} \mathbf{B} \mathbf{u}$ 


\section{Convexity}

$$
\phi(\gamma)=\overbrace{\ln \left|\mathbf{A}_{\gamma}\right|}^{2 .}+\overbrace{\sum_{j} h_{j}\left(\gamma_{j}\right)}^{3 .}+\overbrace{\min _{\mathbf{u}} R(\mathbf{u}, \gamma)}^{1 .}
$$

$\mathbf{A}_{\gamma}=\mathbf{X}^{\top} \mathbf{X}+\mathbf{B}^{\top} \Gamma^{-1} \mathbf{B}, \quad R(\mathbf{u}, \gamma)=\|\mathbf{X} \mathbf{u}-\mathbf{y}\|^{2}+\mathbf{u}^{\top} \mathbf{B}^{\top} \Gamma^{-1} \mathbf{B} \mathbf{u}-2 \boldsymbol{\beta}^{\top} \mathbf{B} \mathbf{u}$

- approximate posterior $\mathbb{Q}(\mathbf{u} \mid \mathcal{D})=\mathcal{N}\left(\mathbf{u}^{*}, \mathbf{A}_{\gamma}^{-1}\right)$ 


\section{Convexity}

$\phi(\gamma)=\overbrace{\ln \left|\mathbf{A}_{\gamma}\right|}^{2 .}+\overbrace{\sum_{j} h_{j}\left(\gamma_{j}\right)}^{3 .}+\overbrace{\min _{\mathbf{u}} R(\mathbf{u}, \gamma)}^{[1 .]}$

$\mathbf{A}_{\gamma}=\mathbf{X}^{\top} \mathbf{X}+\mathbf{B}^{\top} \Gamma^{-1} \mathbf{B}, \quad R(\mathbf{u}, \gamma)=\|\mathbf{X} \mathbf{u}-\mathbf{y}\|^{2}+\mathbf{u}^{\top} \mathbf{B}^{\top} \Gamma^{-1} \mathbf{B} \mathbf{u}-2 \boldsymbol{\beta}^{\top} \mathbf{B} \mathbf{u}$

- approximate posterior $\mathbb{Q}(\mathbf{u} \mid \mathcal{D})=\mathcal{N}\left(\mathbf{u}^{*}, \mathbf{A}_{\gamma}^{-1}\right)$

(1) $R(\mathbf{u}, \gamma)$ jointly convex $\Rightarrow \min _{\mathbf{u}} R(\mathbf{u}, \gamma)$ convex 


\section{Convexity}

$$
\phi(\gamma)=\overbrace{\ln \left|\mathbf{A}_{\gamma}\right|}^{[2 .]}+\overbrace{\sum_{j} h_{j}\left(\gamma_{j}\right)}^{3 .}+\overbrace{\min _{\mathbf{u}} R(\mathbf{u}, \gamma)}^{1 .}
$$

$\mathbf{A}_{\gamma}=\mathbf{X}^{\top} \mathbf{X}+\mathbf{B}^{\top} \Gamma^{-1} \mathbf{B}, \quad R(\mathbf{u}, \gamma)=\|\mathbf{X} \mathbf{u}-\mathbf{y}\|^{2}+\mathbf{u}^{\top} \mathbf{B}^{\top} \Gamma^{-1} \mathbf{B} \mathbf{u}-2 \boldsymbol{\beta}^{\top} \mathbf{B} \mathbf{u}$

- approximate posterior $\mathbb{Q}(\mathbf{u} \mid \mathcal{D})=\mathcal{N}\left(\mathbf{u}^{*}, \mathbf{A}_{\gamma}^{-1}\right)$

(2) $R(\mathbf{u}, \gamma)$ jointly convex $\Rightarrow \min _{\mathbf{u}} R(\mathbf{u}, \gamma)$ convex

(2) $\ln \left|\mathbf{A}_{\gamma}\right|$ convex in $\gamma$ 


\section{Convexity}

$$
\phi(\gamma)=\overbrace{\ln \left|\mathbf{A}_{\gamma}\right|}^{2 .}+\overbrace{\sum_{j} h_{j}\left(\gamma_{j}\right)}^{[3 .]}+\overbrace{\min _{\mathbf{u}} R(\mathbf{u}, \gamma)}^{1 .}
$$

$\mathbf{A}_{\gamma}=\mathbf{X}^{\top} \mathbf{X}+\mathbf{B}^{\top} \Gamma^{-1} \mathbf{B}, \quad R(\mathbf{u}, \gamma)=\|\mathbf{X} \mathbf{u}-\mathbf{y}\|^{2}+\mathbf{u}^{\top} \mathbf{B}^{\top} \Gamma^{-1} \mathbf{B u}-2 \boldsymbol{\beta}^{\top} \mathbf{B u}$

- approximate posterior $\mathbb{Q}(\mathbf{u} \mid \mathcal{D})=\mathcal{N}\left(\mathbf{u}^{*}, \mathbf{A}_{\gamma}^{-1}\right)$

(1) $R(\mathbf{u}, \gamma)$ jointly convex $\Rightarrow \min _{\mathbf{u}} R(\mathbf{u}, \gamma)$ convex

(2) $\ln \left|\mathbf{A}_{\gamma}\right|$ convex in $\gamma$

(3) $h_{j}\left(\gamma_{j}\right)$ convex in $\gamma_{j} \Leftrightarrow \ln t_{j}\left(s_{j}\right)$ concave 


\section{Optimization}

$$
\begin{aligned}
\phi(\gamma)= & \ln |\mathbf{A}|+h(\gamma)+\min _{\mathbf{u}}\left(\|\mathbf{X} \mathbf{u}-\mathbf{y}\|^{2}+\mathbf{s}^{\top} \boldsymbol{\Gamma}^{-1} \mathbf{s}-2 \boldsymbol{\beta}^{\top} \mathbf{s}\right) \\
& \mathbf{A}=\mathbf{X}^{\top} \mathbf{X}+\mathbf{B}^{\top} \boldsymbol{\Gamma}^{-1} \mathbf{B}, \quad \mathbf{s}=\mathbf{B u}
\end{aligned}
$$

- $\phi(\gamma)$ is convex, so things are easy, right?

- gradient hard: $\nabla_{\gamma^{-1}} \ln |\mathrm{A}|=\operatorname{diag}\left(\mathrm{BA}^{-1} \mathrm{~B}^{\top}\right)$

- coordinate descent hard: $n$ linear systems

- coupling term $\ln |\mathrm{A}|$ causing trouble $\Rightarrow$ decouple

- Legendre duality: 


\section{Optimization}

$$
\begin{aligned}
\phi(\gamma)= & \ln |\mathbf{A}|+h(\gamma)+\min _{\mathbf{u}}\left(\|\mathbf{X} \mathbf{u}-\mathbf{y}\|^{2}+\mathbf{s}^{\top} \boldsymbol{\Gamma}^{-1} \mathbf{s}-2 \boldsymbol{\beta}^{\top} \mathbf{s}\right) \\
& \mathbf{A}=\mathbf{X}^{\top} \mathbf{X}+\mathbf{B}^{\top} \boldsymbol{\Gamma}^{-1} \mathbf{B}, \quad \mathbf{s}=\mathbf{B u}
\end{aligned}
$$

- $\phi(\gamma)$ is convex, so things are easy, right?

- gradient hard: $\nabla_{\gamma^{-1}} \ln |\mathbf{A}|=\operatorname{diag}\left(\mathbf{B A}^{-1} \mathbf{B}^{\top}\right)$

- coordinate descent hard: $n$ linear systems

- coupling term $\ln |\mathbf{A}|$ causing trouble $\Rightarrow$ decouple 


\section{Optimization}

$$
\begin{aligned}
\phi(\gamma)= & \ln |\mathbf{A}|+h(\gamma)+\min _{\mathbf{u}}\left(\|\mathbf{X} \mathbf{u}-\mathbf{y}\|^{2}+\mathbf{s}^{\top} \boldsymbol{\Gamma}^{-1} \mathbf{s}-2 \boldsymbol{\beta}^{\top} \mathbf{s}\right) \\
& \mathbf{A}=\mathbf{X}^{\top} \mathbf{X}+\mathbf{B}^{\top} \boldsymbol{\Gamma}^{-1} \mathbf{B}, \quad \mathbf{s}=\mathbf{B u}
\end{aligned}
$$

- $\phi(\gamma)$ is convex, so things are easy, right?

- gradient hard: $\nabla_{\gamma^{-1}} \ln |\mathbf{A}|=\operatorname{diag}\left(\mathbf{B A}^{-1} \mathbf{B}^{\top}\right)$

- coordinate descent hard: $n$ linear systems

- coupling term $\ln |\mathbf{A}|$ causing trouble $\Rightarrow$ decouple

- $\gamma^{-1} \mapsto \ln |\mathbf{A}|$ concave

- Legendre duality:

$$
\ln |\mathbf{A}| \leq \underbrace{\mathbf{z}^{\top}\left(\gamma^{-1}\right)-g^{*}(\mathbf{z})}_{\text {convex in } \gamma}
$$

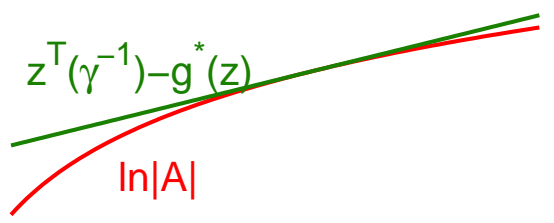




\section{Double Loop Details}

$$
\begin{gathered}
\phi(\gamma)=\ln |\mathbf{A}|+h(\gamma)+\min _{\mathbf{u}}\left(\|\mathbf{X} \mathbf{u}-\mathbf{y}\|^{2}+\mathbf{s}^{\top} \boldsymbol{\Gamma}^{-1} \mathbf{s}-2 \boldsymbol{\beta}^{\top} \mathbf{s}\right) \\
\mathbf{A}=\mathbf{X}^{\top} \mathbf{X}+\mathbf{B}^{\top} \boldsymbol{\Gamma}^{-1} \mathbf{B}, \quad \mathbf{s}=\mathbf{B u}
\end{gathered}
$$

- outer: $\phi=\phi_{\cap}+\phi_{\cup} \leq \phi_{/}+\phi_{\cup}$,

$\ln |\mathbf{A}| \leq \mathbf{z}^{\top}\left(\gamma^{-1}\right)-g^{*}(\mathbf{z})$

- inner: $\min _{\boldsymbol{\gamma}} \phi_{/}+\phi_{\cup}$
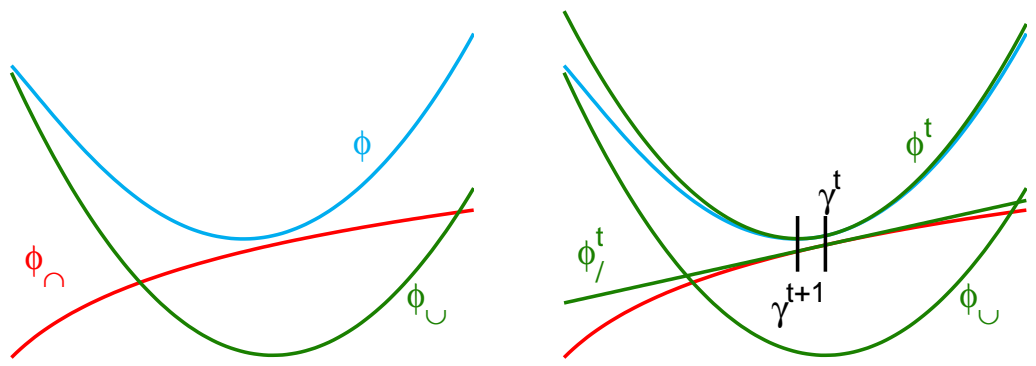

(6) 


\section{Double Loop Summary}

$$
\begin{gathered}
\phi(\gamma)=\ln |\mathbf{A}|+h(\gamma)+\min _{\mathbf{u}}\left(\|\mathbf{X} \mathbf{u}-\mathbf{y}\|^{2}+\mathbf{s}^{\top} \boldsymbol{\Gamma}^{-1} \mathbf{s}-2 \boldsymbol{\beta}^{\top} \mathbf{s}\right) \\
\mathbf{A}=\mathbf{X}^{\top} \mathbf{X}+\mathbf{B}^{\top} \boldsymbol{\Gamma}^{-1} \mathbf{B}, \quad \mathbf{s}=\mathbf{B u}
\end{gathered}
$$

Gradient Based Algorithm

- for $t=1 . . \mathcal{O}(n)$

- compute gradient $\nabla_{\gamma} \phi$

- get descent direction $\delta$

- compute stepsize $\lambda$

- $\gamma^{t+1} \leftarrow \gamma^{t}+\lambda \delta$
Double Loop Algorithm

- for $t=1 . . \mathcal{O}(1)$

- outer loop update: compute gradient $\nabla_{\gamma} \phi$

- inner loop optim: $\gamma^{t+1} \leftarrow \operatorname{IRLS}\left(\gamma^{t}\right)$

- efficient use of expensive computations 


\section{Experimental Design}

- approximate posterior $\mathbb{Q}(\mathbf{u})=\mathcal{N}\left(\mathbf{u}^{*}, \mathbf{A}^{-1}\right)$, conditioned on $\mathcal{D}$

- relative entropy $\mathrm{KL}\left[\mathbb{Q}^{\prime} \| \mathbb{Q}\right]=\mathcal{H}\left[\mathbb{Q}^{\prime} \| \mathbb{Q}\right]-\mathcal{H}\left[\mathbb{Q}^{\prime}\right] \geq 0$

- information gain score

- MRI: continuous Gaussian sites

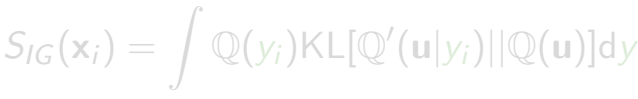

- Classification: binary Bernoulli sites

- design loop: (1) update $\mathbb{Q},(2)$ design decision, (3) measurement 


\section{Experimental Design}

- approximate posterior $\mathbb{Q}(\mathbf{u})=\mathcal{N}\left(\mathbf{u}^{*}, \mathbf{A}^{-1}\right)$, conditioned on $\mathcal{D}$

- relative entropy $\mathrm{KL}\left[\mathbb{Q}^{\prime} \| \mathbb{Q}\right]=\mathcal{H}\left[\mathbb{Q}^{\prime} \| \mathbb{Q}\right]-\mathcal{H}\left[\mathbb{Q}^{\prime}\right] \geq 0$

- information gain score

- MRI: continuous Gaussian sites

$$
S_{I G}\left(\mathbf{x}_{i}\right)=\int \mathbb{Q}\left(y_{i}\right) \mathrm{KL}\left[\mathbb{Q}^{\prime}\left(\mathbf{u} \mid y_{i}\right) \| \mathbb{Q}(\mathbf{u})\right] \mathrm{d} y_{i}
$$

- Classification: binary Bernoulli sites

- design loop: (1) update $\mathbb{Q},(2)$ design decision, (3) measurement 


\section{Experimental Design}

- approximate posterior $\mathbb{Q}(\mathbf{u})=\mathcal{N}\left(\mathbf{u}^{*}, \mathbf{A}^{-1}\right)$, conditioned on $\mathcal{D}$

- relative entropy $\mathrm{KL}\left[\mathbb{Q}^{\prime} \| \mathbb{Q}\right]=\mathcal{H}\left[\mathbb{Q}^{\prime} \| \mathbb{Q}\right]-\mathcal{H}\left[\mathbb{Q}^{\prime}\right] \geq 0$

- information gain score

- MRI: continuous Gaussian sites

$$
S_{I G}\left(\mathbf{x}_{i}\right)=\int \mathbb{Q}\left(y_{i}\right) \mathrm{KL}\left[\mathbb{Q}^{\prime}\left(\mathbf{u} \mid y_{i}\right) \| \mathbb{Q}(\mathbf{u})\right] \mathrm{d} y_{i}
$$

- Classification: binary Bernoulli sites

$$
S_{I G}\left(\mathbf{b}_{j}\right)=\sum_{c_{j}= \pm 1} \mathbb{Q}\left(c_{j}\right) \mathrm{KL}\left[\mathbb{Q}^{\prime}\left(\mathbf{u} \mid c_{j}\right) \| \mathbb{Q}(\mathbf{u})\right]
$$




\section{Experimental Design}

- approximate posterior $\mathbb{Q}(\mathbf{u})=\mathcal{N}\left(\mathbf{u}^{*}, \mathbf{A}^{-1}\right)$, conditioned on $\mathcal{D}$

- relative entropy $\mathrm{KL}\left[\mathbb{Q}^{\prime} \| \mathbb{Q}\right]=\mathcal{H}\left[\mathbb{Q}^{\prime} \| \mathbb{Q}\right]-\mathcal{H}\left[\mathbb{Q}^{\prime}\right] \geq 0$

- information gain score

- MRI: continuous Gaussian sites

$$
S_{I G}\left(\mathbf{x}_{i}\right)=\int \mathbb{Q}\left(y_{i}\right) \mathrm{KL}\left[\mathbb{Q}^{\prime}\left(\mathbf{u} \mid y_{i}\right) \| \mathbb{Q}(\mathbf{u})\right] \mathrm{d} y_{i}
$$

- Classification: binary Bernoulli sites

$$
S_{I G}\left(\mathbf{b}_{j}\right)=\sum_{c_{j}= \pm 1} \mathbb{Q}\left(c_{j}\right) \mathrm{KL}\left[\mathbb{Q}^{\prime}\left(\mathbf{u} \mid c_{j}\right) \| \mathbb{Q}(\mathbf{u})\right]
$$

- design loop: (1) update $\mathbb{Q},(2)$ design decision, (3) measurement 


\section{Experiments}

- large scale active learning using approximate inference
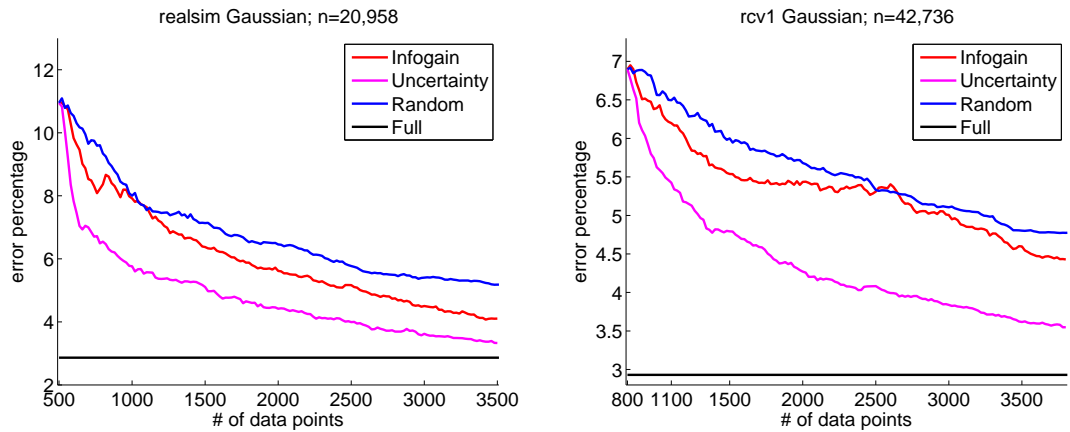


\section{Conclusions}

- variational relaxation convex iff. MAP estimation convex

- posterior approximation successfully drives experimental design

- fully scalable and generic double loop algorithm

- computational primitives: CG (means), Lanczos (variances)

- complexity $=$ speed of MVMs with $\mathbf{X}$ and $\mathbf{B}$ 


\section{Appendix: Convexity of log determinant}

\section{Theorem}

$\gamma \mapsto \ln \left|\mathbf{A}_{\gamma}\right|$ is convex. $\mathbf{A}_{\gamma}=\mathbf{X}^{\top} \mathbf{X}+\mathbf{B}^{\top} \Gamma^{-1} \mathbf{B}$

- $(\mathbf{u}, \gamma) \mapsto \mathbf{u}^{\top}\left(\mathbf{X}^{\top} \mathbf{X}+\mathbf{B}^{\top} \Gamma^{-1} \mathbf{B}\right) \mathbf{u}$ jointly convex

- $(\mathbf{u}, \gamma) \mapsto \exp \left(-\frac{1}{2} \mathbf{u}^{\top} \mathbf{A}_{\gamma} \mathbf{u}\right)$ jointly log-concave

- marginalization theorem (Prékopa)

- Log-concave functions are closed under marginalization.

- Gaussian integral: $\ln \left|\mathbf{A}_{\gamma}\right|=n \ln 2 \pi-2 \ln \int \exp \left(-\frac{1}{2} \mathbf{u}^{\top} \mathbf{A}_{\gamma} \mathbf{u}\right) \mathrm{d} \mathbf{u}$

\section{Theorem}

$\gamma \mapsto \ln \mid \mathbf{X}^{\top} \mathbf{X}+\mathbf{B}^{\top} f(\ulcorner) \mathbf{B} \mid$ is convex iff. $\ln f(\gamma)$ is convex. 


\section{Appendix: Convexity of individual height functions}

\section{Theorem}

$h(\gamma)$ is convex iff. $g(s)=\ln t(s)$ is concave in $s$ and convex in $x=s^{2}$.

(1) $t(s)=\max _{\gamma} \geq 0 \exp \left(-\frac{s^{2}}{2 \gamma}-h(\gamma)\right), h(\gamma)=g^{*}\left(-\frac{1}{2 \gamma}\right)$

(2) $h(\gamma)=\max _{s \geq 0} f(s, \gamma)=\max _{s \geq 0} \frac{-1}{2 \gamma} s^{2}-g(s)=\max _{x} \geq 0 \frac{-1}{2 \gamma} x-g(x)$

(3) $0 \stackrel{!}{=} \frac{\partial}{\partial x} f(x, \gamma) \Rightarrow g^{\prime}\left(x_{*}\right)=-\frac{1}{2 \gamma}$ implicitly defining $x_{*}(\gamma)$ and $h(\gamma)=f\left(x_{*}(\gamma), \gamma\right)$

(3) $\frac{\mathrm{d} x_{*}}{\mathrm{~d} \gamma}=\frac{1}{2 \gamma^{2} g^{\prime \prime}\left(x_{*}\right)} \wedge x \mapsto g(x)$ convex $\Rightarrow \gamma \mapsto x_{*}$ increasing $\Rightarrow \gamma \mapsto s_{*}$ increasing

(3) $0 \stackrel{!}{=} \frac{\partial}{\partial s} f(s, \gamma) \Rightarrow g^{\prime}\left(s_{*}\right)=-\frac{s_{*}}{\gamma}, \quad g(s)$ concave $\Rightarrow g^{\prime}(s)$ decreasing

(6) $h^{\prime}(\gamma)=\frac{\partial}{\partial \gamma} f\left(s_{*}, \gamma\right)=\frac{1}{2}\left(g^{\prime}\left(s_{*}\right)\right)^{2} \wedge(5) \Rightarrow s_{*} \mapsto h^{\prime}(\gamma)$ increasing due to square

(1) $(4,6) \Rightarrow \gamma \mapsto h^{\prime}(\gamma)$ increasing $\Rightarrow h(\gamma)$ convex 